\title{
VÍTIMAS DE VIOLÊNCIA SEXUAL ATENDIDAS EM UM SERVIÇO DE REFERÊNCIA
}

Tatiane Herreira Trigueiro', Miriam Aparecida Barbosa Merighi², Adeli Regina Prizybicien de Medeiros ${ }^{3}$, Cléa Elisa Lopes Ribeiro ${ }^{4}$, Nely Dayse Santos da Mata ${ }^{1}$, Maria Cristina Pinto de Jesus ${ }^{5}$

${ }^{1}$ Enfermeira. Doutoranda em Enfermagem. Universidade de São Paulo. São Paulo-SP-Brasil.

${ }^{2}$ Enfermeira. Doutora em Enfermagem. Professora da Universidade de São Paulo. São Paulo-SP-Brasil.

${ }^{3}$ Enfermeira. Mestre em Enfermagem. Hospital Universitário da Universidade Federal do Paraná. Curitiba-PR-Brasil.

${ }^{4}$ Médica. Mestre em Medicina Interna. Hospital Universitário da Universidade Federal do Paraná. Curitiba-PR-Brasil.

${ }^{5}$ Enfermeira. Doutora em Enfermagem. Professora da Universidade Federal de Juiz de Fora. Juiz de Fora-MG-Brasil.

RESUMO: Objetivou-se conhecer aspectos da violência sexual e adesão das vítimas ao seguimento ambulatorial. Estudo transversal, com coleta retrospectiva de dados em um serviço de referência no estado do Paraná. A coleta foi realizada de fevereiro a maio de 2014 mediante fichas de notificação de 2009 a 2013. Foram atendidos 1272 agredidos sexualmente, sendo 53,46\% deles com idade entre 12 e 18 anos e 94,65\%, mulheres. Em mais da metade dos casos, o agressor era desconhecido (53,38\%), a agressão ocorreu nas residências (39,30\%) e vias públicas (35\%), à noite (50,33\%) e, em $96,67 \%$ dos casos, houve penetração vaginal. A indicação da Profilaxia Pós-Exposição às vítimas ocorreu em 76,4\% e a contracepção de emergência foi administrada em $64,77 \%$ das mulheres. Apenas 19,54\% completaram o seguimento ambulatorial. As evidências indicam a necessidade de estratégias de atendimento que motivem a vítima de violência sexual a finalizar o tratamento. DESCRITORES: Violência sexual; Violência contra a mulher; Enfermagem.

\section{VICTIMS OF SEXUAL VIOLENCE ATTENDED IN A SPECIALIZED SERVICE}

\begin{abstract}
The aim was to investigate aspects of sexual violence and the victims' adherence to outpatient followup. This transversal study involved retrospective data collection in a specialized service in the Brazilian state of Paraná. The collection was undertaken between February and May 2014 from the notification files of 2009 - 2013. A total of 1,272 persons who had been the victims of sexual assault was attended; $53.46 \%$ of them were aged between 12 and 18 years old, and $94.65 \%$ were female. In more than half of the cases, the aggressor was unknown (53.38\%), the aggression took place in homes (39.30\%) and on public highways (35\%), at night (50.33\%) and, in $96.67 \%$ of cases, there was vaginal penetration. Indication of PostExposure Prophylaxis to the victims took place in $76.4 \%$ of cases and emergency contraception was administered to $64.77 \%$ of the women. Only $19.54 \%$ completed the outpatient follow-up. The evidence indicates the need for attendance strategies which motivate the victim of sexual violence to complete treatment.
\end{abstract}

DESCRIPTORS: Sexual violence; Violence against women; Nursing.

\section{VÍCTIMAS DE VIOLENCIA SEXUAL ATENDIDAS EN UN SERVICIO DE REFERENCIA}

RESUMEN: El objetivo del estudio fue conocer aspectos de la violencia sexual y adhesión de las víctimas al procedimiento ambulatorial. Estudio transversal cuyos datos fueron obtenidos de modo retrospectivo en un servicio de referencia en el estado de Paraná. Los datos fueron recogidos de febrero a mayo de 2014 por medio de fichas de notificación de 2009 a 2013. Fueron atendidos 1272 agredidos sexualmente, siendo la edad de $53,46 \%$ de ellos entre 12 y 18 años y 94,65\%, mujeres. En más de la mitad de los casos, el agresor era desconocido (53,38\%), la agresión ocurrió en las residencias $(39,30 \%$ ) y locales públicos (35\%), por la noche $(50,33 \%)$ y, en $96,67 \%$ de los casos, hubo penetración vaginal. La indicación de la Profilaxia Pos Exposicón a las víctimas ocurrió en 76,4\% y la contracepción de emergencia fue administrada en $64,77 \%$ de las mujeres. Solamente $19,54 \%$ completaron el procedimiento ambulatorial. Las evidencias apuntan la necesidad de estrategias de atendimiento que ayuden la víctima de violencia sexual a finalizar el tratamiento.

DESCRIPTORES: Violencia sexual; Violencia contra la mujer; Enfermería.
Autor Correspondente:

Maria Cristina Pinto de Jesus

Universidade Federal de Juiz de Fora

Rua Barão de Cataguases, 303 - 36015-370 - Juiz de Fora-MG-Brasil

E-mail: mariacristina.jesus@ufjf.edu.br
Recebido: 18/03/2015

Finalizado: 11/05/2015 


\section{INTRODUÇÃO}

A violência sexual tem sido objeto de preocupação em todo o mundo, uma vez que pode trazer consequências amplificadas à saúde biopsicossocial da vítima. Um agravante é que, em muitos casos, o agressor é o companheiro ou familiar. Estudo realizado pela Organização Mundial de Saúde (OMS) mostrou que a prevalência de mulheres que relataram ter sofrido pelo menos um episódio de violência sexual na vida pelo parceiro íntimo foi maior na Etiópia (58,6\%), seguida por Bangladesh (49,7\%) e Peru $(46,7 \%)$. O Brasil teve prevalência de $14,3 \%$, e o país com a menor taxa foi o Japão $(6,2 \%)^{(1)}$.

Outra questão relevante é que a principal vítima tem sido a mulher. Pesquisa realizada no Instituto Médico Legal da cidade de Ucayali, Peru, evidenciou a ocorrência de 110 atendimentos decorrentes de atentados de ordem sexual e, destes, todas as vítimas foram mulheres. Em $52,8 \%$ dos casos, as mulheres tinham idades entre 13 e 18 anos e 12,8\%, mais de 18 anos; $35 \%$ referiram ter sofrido violência sexual com penetração vaginal e $25,2 \%$ referiram que sofreram atos libidinosos ${ }^{(2)}$.

No Brasil, uma investigação epidemiológica realizada pela Vigilância de Violências e Acidentes (VIVA) em 136 serviços de urgência e emergência constatou que foram realizados 8.766 atendimentos por agravos decorrentes de violência em suas formas doméstica, sexual e outras. Desse total, 2.780 casos foram registrados como violência sexual, sendo 2.429 cometidos contra mulheres ${ }^{(3)}$.

No Paraná, entre os anos de 2009 e 2010, o número de casos de violência notificados chegou a 10.515. Entre estes, $70 \%$ foram cometidos contra mulheres e o maior município notificador foi Curitiba com 1.514 casos somente no ano de 2010. Entre as 10.515 notificações, 21,87\% foram registradas como violência sexual( ${ }^{(4)}$.

Os serviços especializados às mulheres vítimas de violência sexual seguem o modelo de atenção multidisciplinar e interdepartamental, baseado no protocolo de atendimento preconizado pelo Ministério da Saúde, que estabelece o mínimo de quatro consultas para a mulher após a procura pelo serviço, resultando em, no mínimo, 180 dias de seguimento ambulatorial com médicos, enfermeiros, psicólogos e assistentes sociais, realização de exames e tratamento medicamentoso ${ }^{(5)}$. Apesar da disponibilidade do serviço, recomendação para a notificação da agressão e procura pelo serviço especializado, nem sempre isso ocorre.

Uma das dificuldades de atendimento às pessoas em situação de violência sexual, apontada em um estudo realizado em Fortaleza, estado do Ceará, foi a barreira formada pela própria vítima que, geralmente, vem acompanhada de vergonha, constrangimento, insegurança, medo, sendo este referido como o principal empecilho ao atendimento ${ }^{(6)}$.

Ainda que não seja factível avaliar o impacto da violência sexual no sofrimento psíquico e o desenvolvimento ou não de sintomas em mulheres, é possível inferir que muitas das vítimas necessitariam de apoio em saúde mental e, sem utilizarem o acompanhamento ambulatorial oferecido, podem desenvolver sequelas de longa duração ${ }^{(7)}$.

Frente ao exposto, as seguintes questões nortearam este estudo: quais as características da violência sexual impetrada contra vítimas atendidas em um serviço de referência do estado do Paraná? No período estudado, as vítimas de violência sexual aderiram ao seguimento ambulatorial preconizado pelo Ministério da Saúde?

Objetivou-se conhecer aspectos relacionados à violência sexual e adesão das vítimas ao seguimento ambulatorial preconizado pelo Ministério da Saúde.

A contribuição do estudo consiste em apresentar evidências que poderão subsidiar a elaboração de estratégias de atendimento de profissionais de saúde a vítimas de violência sexual, com vistas a estimular a maior adesão destas ao seguimento ambulatorial.

\section{MÉTODO}

Trata-se de um estudo descritivo de corte transversal, com coleta retrospectiva de dados secundários de um hospital universitário da capital paranaense, referência para vítimas de violência.

A coleta de dados foi realizada no período de fevereiro a maio de 2014 mediante análise das fichas de notificação da violência doméstica, sexual e outras violências interpessoais preconizadas pelo Ministério da Saúde ocorridas no período de 
2009 a $2013^{(8)}$, disponíveis no Sistema Nacional de Informações sobre Agravos de Notificação (SINAN) do próprio hospital. Os dados foram fornecidos pelo hospital em planilha Excel 2007 e continham informações sobre número da ficha de notificação, ano do atendimento, sexo, idade, escolaridade, situação conjugal, agressor, município, local e horário da ocorrência, tipo de violência sexual, indicação da administração do antirretroviral, gestação resultante da agressão sexual e indicação da administração da contracepção de emergência e abortamento.

Como critérios de inclusão utilizaram-se todas as fichas de notificação-investigação individual, referentes à violência sexual sofrida por pessoas que procuraram o serviço de referência do hospital, entre os anos de 2009 a 2013, que continham informações que possibilitassem a coleta de dados a respeito da agressão sexual sofrida. Não houve exclusão de nenhuma ficha de notificação.

Para o registro das informações foi consultado o sistema informatizado do hospital, que subsidiou a construção de um instrumento para registro de presenças e ausências no ambulatório de infectologia. $\mathrm{O}$ encaminhamento para este ambulatório é feito quando o tempo entre a violência e o atendimento é inferior a 72 horas, e quando não se trata de agressão crônica. No primeiro atendimento, o enfermeiro realiza o acolhimento da vítima e solicita a presença do médico perito do Instituto Médico Legal e do médico residente da área de ginecologia para atendimento clínico. Nessa oportunidade são realizados os exames e administrados os medicamentos para evitar a ocorrência de Doenças Sexualmente Transmissíveis (DSTs) e gestação decorrentes da violência. Se houver indicação clínica: contato com mucosas, abuso sexual não crônico e tempo decorrido entre a violência e o primeiro atendimento $<72$ horas é iniciada a administração da Profilaxia Pós Exposição (PEP) para o HIV, por um período de 28 dias $^{(4)}$.

Após a avaliação do primeiro atendimento, aquelas mulheres que tiverem indicação são orientadas e encaminhadas para os retornos subsequentes ao serviço de infectologia, em torno de 10, 30, 90 e 180 dias após a violência. Nas consultas subsequentes são requisitados exames preconizados em protocolo, realizadas orientações e acompanhamento de adesão à PEP. Na consulta de 90 dias, o serviço oferece a oportunidade à mulher de voltar após três meses para a consulta de 180 dias ou receber os resultados dos exames por via telefônica. Além dessas possibilidades a mulher é encaminhada para atendimento psicológico e ao serviço social(9). A adesão ao seguimento ambulatorial se configura mediante o comparecimento às quatro consultas estabelecidas, a utilização dos medicamentos prescritos e a concordância com a realização dos exames previstos em protocolo ${ }^{(5)}$.

Os dados foram analisados com auxílio do Programa Excel 2007, versão 12.0, por meio de estatística descritiva. Verificaram-se o total e a média das variáveis correspondentes às características das vítimas, da violência e ainda a adesão ao seguimento ambulatorial cujos valores são apresentados em tabelas e quadro. O projeto de pesquisa foi aprovado pelo Comitê de Ética em Pesquisa, conforme Parecer $n^{\circ}$ 507.604, de sete de janeiro de 2014.

\section{RESULTADOS}

Foram notificadas como suspeita de violência sexual 1272 pessoas no período de 2009 a 2013, sendo constatado no ano de 2009 um total de 142 notificações, em 2010 houve 253, 268 em 2011, 270 em 2012 e em 2013 houve 339 notificações.

Conforme a Tabela 1, quase todas as pessoas atendidas eram mulheres, 1.204 (94,65\%). Apesar de o serviço ser caracterizado como de atendimento à pessoa adulta, constatouse a presença de $29(2,28 \%)$ crianças com idade inferior a 12 anos. O maior número de atendimentos ocorreu na faixa etária de 12 aos 18 anos, 680 (53,46\%), sendo a idade máxima registrada de 81 anos. Quanto à situação conjugal, a maioria se declarou solteira, 942 (74,05\%), e, no quesito escolaridade, mais da metade tinha ensino fundamental completo (51,65\%).

A Tabela 2 mostra que a maioria das vítimas não conhecia o agressor (53,38\%). Quanto ao local, 637 (50\%) casos ocorreram na região metropolitana da capital paranaense, sendo que 500 (39,30\%) aconteceram nas residências, seguidos das vias públicas em 445 casos (35\%). Quanto ao horário da agressão sexual, 607 $(47,72 \%)$ ocorrências aconteceram entre as 19 horas e as 7 horas do dia seguinte. Em 1.164 casos de agressão sexual a mulheres, ocorreu penetração via vaginal. 
Constatou-se o registro de 81 (6,36\%) casos de gestação, sendo 55 (4,32\%) decorrentes da agressão sexual sofrida.

A contracepção de emergência foi administrada em $824(64,77 \%)$ dos casos e a indicação da PEP foi realizada em $973(76,4 \%)$ das fichas de notificação. A partir desta indicação, as 973 vítimas foram encaminhadas para seguimento ambulatorial do serviço de infectologia, somente 394 (40,5\%)

Tabela 1 - Características das vítimas de violência sexual. Curitiba-PR-Brasil, 2014

\begin{tabular}{lcc}
\hline Variáveis & $\mathbf{N}$ & $\%$ \\
\hline Sexo & & \\
\hline Feminino & 1.204 & 94,65 \\
\hline Masculino & 68 & 5,35 \\
\hline Faixa etária & & \\
\hline$<12$ anos & 29 & 2,28 \\
\hline 12 a 18 anos & 680 & 53,46 \\
\hline 19 a 59 anos & 553 & 43,47 \\
\hline$\geq 60$ anos & 10 & 0,79 \\
\hline Situação conjugal & & \\
\hline Solteiros & 942 & 74,05 \\
\hline Casados/União estável & 169 & 13,29 \\
\hline Viúvos & 16 & 1,26 \\
\hline Separados & 79 & 6,21 \\
\hline Campo ignorado & 66 & 5,19 \\
\hline Escolaridade & & \\
\hline Sem escolaridade & 11 & 0,86 \\
\hline Fundamental & 459 & 36,09 \\
\hline incompleto & 133 & 10,45 \\
\hline Fundamental completo & 240 & 18,86 \\
\hline Médio incompleto & 151 & 11,89 \\
\hline Médio completo & 83 & 6,52 \\
\hline Superior incompleto & 50 & 3,93 \\
\hline Superior completo & 145 & 11,40 \\
\hline Campo ignorado & & \\
\hline
\end{tabular}

Fonte: Sistema de Informação de Agravos de Notificação. Serviço de Epidemiologia. Hospital de Clínicas. Paraná, Brasil, 2014. compareceram ao primeiro atendimento.

Observa-se, na Tabela 3 que do total de 394 vítimas da violência sexual que compareceram ao primeiro atendimento apenas 77 (19,54\%) estavam presentes no quarto retorno, finalizando o tratamento com 180 dias de acompanhamento. Salienta-se que em 2012, somente oito vítimas das 89 que iniciaram o primeiro atendimento, finalizaram o tratamento.

Tabela 2 - Características da violência sexual. Curitiba-PR-Brasil, 2014

\begin{tabular}{|c|c|c|}
\hline Variáveis & $\mathbf{N}$ & $\%$ \\
\hline \multicolumn{3}{|l|}{ Agressor } \\
\hline Pai & 34 & 2,70 \\
\hline Mãe & 13 & 1,02 \\
\hline Padrasto & 45 & 3,54 \\
\hline Cônjuge/namorado & 94 & 7,39 \\
\hline Ex-cônjuge/namorado & 44 & 3,45 \\
\hline Filho & 01 & 0,07 \\
\hline Desconhecido & 679 & 53,38 \\
\hline Irmão & 08 & 0,63 \\
\hline Conhecido & 318 & 25 \\
\hline Cuidador & 02 & 0,16 \\
\hline $\begin{array}{l}\text { Patrão/relação } \\
\text { Institucional }\end{array}$ & 12 & 0,94 \\
\hline Policial/agente da Lei & 02 & 0,15 \\
\hline Outros & 20 & 1,57 \\
\hline \multicolumn{3}{|l|}{ Local de ocorrência } \\
\hline Residência & 500 & 39,30 \\
\hline Habitação coletiva & 10 & 0,78 \\
\hline Escola & 11 & 0,86 \\
\hline $\begin{array}{l}\text { Local de prática } \\
\text { esportiva }\end{array}$ & 15 & 1,18 \\
\hline Bar ou similar & 14 & 1,10 \\
\hline Via pública & 445 & 35 \\
\hline Comércio & 24 & 1,9 \\
\hline Indústria/construção & 5 & 0,39 \\
\hline Outros & 199 & 15,64 \\
\hline Campo ignorado & 49 & 3,85 \\
\hline \multicolumn{3}{|l|}{ Horário } \\
\hline Entre $19 \mathrm{~h}$ e $7 \mathrm{~h}$ & 607 & 47,72 \\
\hline Entre $7 \mathrm{~h}$ e $19 \mathrm{~h}$ & 387 & 30,42 \\
\hline Não registrado & 278 & 21,86 \\
\hline \multicolumn{3}{|l|}{ Tipo de agressão sexual } \\
\hline Penetração vaginal & 1164 & 91,50 \\
\hline Outros & 108 & 8,50 \\
\hline
\end{tabular}

Fonte: Sistema de Informação de Agravos de Notificação. Serviço de Epidemiologia. Hospital de Clínicas. Paraná, Brasil, 2014. 
Tabela 3 - Adesão ao seguimento ambulatorial para vítimas de violência sexual, período 2009-2013. CuritibaPR-Brasil, 2014

\begin{tabular}{|c|c|c|c|c|c|c|c|c|c|c|}
\hline \multirow[t]{2}{*}{ Ano } & \multicolumn{2}{|c|}{$\begin{array}{c}\text { Primeiro } \\
\text { atendimento }\end{array}$} & \multicolumn{2}{|c|}{$\begin{array}{c}\text { Primeiro retorno } \\
\text { (10 dias) }\end{array}$} & \multicolumn{2}{|c|}{$\begin{array}{l}\text { Segundo retorno } \\
\text { (30 dias) }\end{array}$} & \multicolumn{2}{|c|}{$\begin{array}{l}\text { Terceiro retorno } \\
\text { (90 dias) }\end{array}$} & \multicolumn{2}{|c|}{$\begin{array}{l}\text { Quarto retorno } \\
\text { (180 dias) }\end{array}$} \\
\hline & $\mathbf{n}$ & $\%$ & $n$ & $\%$ & $\mathbf{n}$ & $\%$ & $\mathbf{n}$ & $\%$ & $n$ & $\%$ \\
\hline 2009 & 48 & 12,18 & 33 & 14,47 & 10 & 7,64 & 12 & 8,63 & 11 & 14,29 \\
\hline 2010 & 93 & 23,60 & 39 & 17,11 & 22 & 16,79 & 34 & 24,46 & 20 & 25,98 \\
\hline 2011 & 69 & 17,51 & 58 & 25,44 & 23 & 17,55 & 24 & 17,27 & 22 & 28,57 \\
\hline 2012 & 89 & 22,59 & 40 & 17,54 & 37 & 28,25 & 28 & 20,14 & 8 & 10,38 \\
\hline 2013 & 95 & 24,12 & 58 & 25,44 & 39 & 29,77 & 41 & 29,50 & 16 & 20,78 \\
\hline Total & 394 & 100 & 228 & 100 & 131 & 100 & 139 & 100 & 77 & 100 \\
\hline
\end{tabular}

Fonte: Sistema de Informação de Agravos de Notificação. Serviço de Epidemiologia. Hospital de Clínicas. Paraná, Brasil, 2014.

\section{DISCUSSÃO}

O número anual crescente de atendimentos às vítimas de violência sexual constatado evidencia o aumento da notificação dos casos no cenário estudado. Este é um dado relevante, considerando que, de acordo com a literatura, a violência, em especial a do tipo sexual no mundo tem elevada subnotificação ${ }^{(9)}$.

Apesar disso, a violência sexual, embora seja um fenômeno de grande magnitude e impacto na saúde da mulher, permanece oculta na sociedade, pois ainda é reduzido número de vítimas que realizam a denúncia. Salienta-se que o ato de notificar inicia um processo que visa interromper as atitudes e comportamentos violentos por parte de qualquer agressor ${ }^{(10)}$.

Em conformidade com a maioria dos estudos sobre violência sexual, o elevado percentual de mulheres submetidas à agressão sexual apresentado nesta pesquisa confirma a vulnerabilidade da mulher que vivencia esta situação.

No que tange à idade das vítimas no momento da agressão sexual, os resultados desta pesquisa corroboram os dados apresentados por outras investigações, inclusive os do estudo realizado em Campina Grande, estado da Paraíba, cuja idade variou de 12 a 18 anos, com média de 16 anos $^{(11)}$.

O fato de a maioria dos participantes desta pesquisa ter cursado até o ensino fundamental completo se aproxima de um estudo realizado em Fortaleza, estado do Ceará, no Centro Estadual de Referência para Vítimas de Violência que identificou cerca de $62 \%$ das mulheres atendidas com baixa escolaridade $\mathrm{e}^{(12)}$, em Campinas, estado de São Paulo, com 42,4\% dos 664 casos de violência sexual analisados que tinham até 8 anos de escolaridade ${ }^{(7)}$.

No presente estudo, a maioria das vítimas declarou que o agressor foi uma pessoa desconhecida. Este resultado também foi encontrado em estudo realizado no Rio Grande do Sul, cujo percentual de casos foi de $53,9 \%{ }^{(13)}$.

Contrapondo estas evidências, pesquisa realizada em Campina Grande, estado da Paraíba, mostrou que $78,9 \%$ das mulheres vítimas de violência sexual classificaram o agressor como conhecido, pessoa com quem conversavam, tinham o hábito de cumprimentar ou sabiam o nome, sem parentesco familiar ${ }^{(11)}$. Também, uma investigação realizada em um centro de atendimento a vítimas de violência sexual na Cidade do Cabo, na África do Sul, constatou que, das 135 participantes da pesquisa, 46\% declararam que os agressores eram vizinhos, amigos ou ex-namorados ${ }^{(14)}$.

O maior percentual de ocorrências de agressões sexuais, encontrado no presente estudo, foi na região metropolitana da capital paranaense, nas residências ou vias públicas. Este resultado também foi encontrado em pesquisa realizada em um Centro de Atendimento de Campinas, estado de São Paulo, com aproximadamente 54\% dos eventos violentos em vias públicas e $46 \%$ na residência da vítima ${ }^{(15)}$.

Quanto ao horário em que a vítima sofreu a violência sexual, neste estudo, prevaleceu o noturno, assim como nos estudos realizados em Campinas, em 2011 (56,8\% dos casos) $\mathrm{e}^{(15)}$, em 2013, quando a situação violenta ocorreu entre 18h e 7 horas em $75,9 \%$ dos $\operatorname{casos}^{(7)}$.

$\mathrm{O}$ alto percentual de agressão sexual com penetração vaginal constatado neste estudo também foi verificado em pesquisa realizada em 
Campinas, com 88,8\% dos $\operatorname{casos}^{(7)}$. Também, o estudo realizado no Sul do Brasil apontou que a forma de violência sexual mais prevalente foi o estupro e a tentativa de estupro com $76,9 \%$ dos casos e 17,9\%, respectivamente ${ }^{(13)}$. Estes dados revelam crime contra o direito da mulher, constituindo-se um grave problema de saúde pública, tanto pela elevada incidência quanto pelas consequências emocionais e físicas à saúde da mulher ${ }^{(16)}$.

Chama-se a atenção para a notificação de 55 casos de gestação decorrente da agressão sexual, a despeito da assistência especializada prestada às mulheres e da oferta da contracepção de emergência. Salienta-se que o hospital onde a pesquisa foi realizada é referência para realização de aborto legal em casos de violência sexual.

No Brasil, a autorização para o aborto nos casos de gravidez resultante de abuso sexual consta da Norma Técnica sobre Prevenção e Tratamento dos Agravos resultantes de Violência Sexual contra Mulheres e Adolescentes, que traz a legislação constante do Código Penal Brasileiro, assim como a necessidade de esclarecer a vítima do direito e da possibilidade de manter a gestação até o seu término(5).

A contracepção de emergência foi realizada em mais de $60 \%$ das mulheres incluídas neste estudo. Tal evidência pode estar relacionada ao fato de os maiores percentuais de vítimas situarem-se nas faixas etárias de 12 a 18 anos $(53,46 \%)$ e 19 a 59 anos (43,47\%), o que totaliza $96,93 \%$ das mulheres, cuja maioria vivencia a fase reprodutiva.

Quando a agressão sexual ocorre durante a vida reprodutiva, a mulher fica sob risco de gravidez decorrente dela. Esse risco varia entre 0,5 e $5 \%$ considerando a aleatoriedade da violência em relação ao período do ciclo menstrual, e se a violência foi um caso isolado ou é continuada. A gravidez decorrente de violência sexual representa, para a maioria das mulheres, uma segunda forma de violência ${ }^{(5)}$. Essa situação pode ser evitada, em muitos casos, com a utilização da contracepção de emergência.

A indicação PEP ao HIV é feita nos casos de violência sexual que envolveu contato com mucosas anal, vaginal e oral. A OMS recomenda que a mulher agredida seja encaminhada ao serviço de saúde em até 72 horas para o início do tratamento, visto que, após esse período, não existem evidências que suportem sua indicação $^{(5)}$. Nesta pesquisa, a PEP foi indicada em, aproximadamente $76 \%$ dos casos, dado semelhante ao encontrado em estudo realizado no Hospital da Mulher em Campinas, com $84 \%$ das vítimas em prescrição da terapia antirretroviral ${ }^{(7)}$.

Salienta-se o registro de 26 mulheres que estavam grávidas por ocasião da violência sexual. Nesse caso, a anticoncepção de emergência é absolutamente contraindicada. Por outro lado, a gravidez, em qualquer idade gestacional, não contraindica o esquema profilático recomendado, exceto o uso da ciprofloxacina durante toda a gestação e do metronidazol que deve ser evitado durante o primeiro trimestre da gravide $z^{(5)}$.

Apesar da importância do acompanhamento da mulher que sofreu a violência sexual, nem sempre as vítimas procuram os serviços de apoio e permanecem até a alta. Em um centro de atendimento a vítimas de violência sexual na Cidade do Cabo, na África do Sul, dos 135 atendimentos, 131 (97,03\%) pessoas deram início ao PEP, contudo 44 não continuaram o tratamento, por esquecimento e/ou por estarem longe de casa ${ }^{(14)}$.

Uma revisão sistemática realizada em Londres que avaliou 24 estudos mundiais sobre a adesão à PEP para HIV em vítimas de violência sexual constatou que a proporção de pessoas que aderiram ao tratamento foi de $40,3 \%$, e o percentual geral de pacientes faltosos aos cuidados foi de $41,2 \%$. Tais evidências confirmam que a baixa taxa de adesão à profilaxia entre as vítimas de violência sexual é um problema global ${ }^{(17)}$.

A baixa taxa de adesão ao seguimento ambulatorial de vítimas de violência sexual encontrada neste estudo (19,54\%) assemelha-se à de outros estudos brasileiros, como o realizado em Campinas, estado de São Paulo. Em 687 atendimentos analisados no período de junho de 2006 a dezembro de 2010 a taxa de abandono do seguimento ambulatorial foi de $24,5 \%^{(7)}$. Outro estudo realizado nesse mesmo município registrou 644 atendimentos, entre os anos de 2000 e 2006, e constatou que 180 mulheres abandonaram o seguimento ambulatorial após a primeira consulta e 67 após a segunda ${ }^{(15)}$.

O número reduzido de vítimas que finalizaram o seguimento ambulatorial (19,54\%) evidenciado no presente estudo, constitui-se uma problemática a ser enfrentada pelos serviços e profissionais de 
saúde. Salienta-se que os serviços de referência nem sempre apresentam recursos humanos e materiais necessários, assim como articulação com a rede de saúde suficientemente adequada para $\mathrm{o}$ atendimento das vítimas de violência sexual ${ }^{(6)}$.

\section{CONCLUSÃO}

Destacam-se como resultados encontrados neste estudo o número crescente de atendimentos/ ano de vítimas de violência sexual na capital paranaense, cuja maioria é constituída de mulheres em idade reprodutiva. Se, por um lado, o crescimento do número de atendimentos retrata o aumento dos casos de agressão que são denunciados e a procura pelos serviços especializados, o fato de o alvo da violência ser a mulher alerta, especialmente os profissionais que atuam nos serviços de saúde, para a importância de atentar para os princípios do cuidado humanizado a ser oferecido a essa clientela.

Outro resultado que merece destaque é o número reduzido de vítimas que tiveram adesão total ao tratamento, finalizando com 180 dias o acompanhamento de saúde. Esta evidência remete à reflexão acerca do risco de saúde a que estas pessoas estão submetidas, considerando os agravos decorrentes da violência sexual e a responsabilidade do profissional de saúde no acolhimento e suporte da vítima.

O estudo apresentou limitações quanto ao fato de utilizar dados secundários retirados de fichas de notificação com informações incompletas. Porém, tais limitações não invalidam as evidências obtidas já que estas proporcionam a reflexão acerca da importância de se pensar estratégias que motivem a vítima de violência sexual a dar continuidade ao seguimento ambulatorial até finalizar o tratamento.

Sugere-se que investigações qualitativas sejam realizadas com este público, com a finalidade de compreender os motivos do abandono do seguimento ambulatorial nos serviços de referência para vítimas da violência.

\section{REFERÊNCIAS}

1. Organização Mundial da Saúde (OMS). Prevenção da violência sexual e da violência pelo parceiro íntimo contra a mulher: ação e produção de evidência. [Internet] 2010 [acesso em 28 out 2014]. Disponível: http://apps.who.int/iris/ bitstream/10665/44350/3/9789275716359_por.pdf

2. Flores PCL. Análisis epidemiológico de lós delitos contra la libertad sexual y la valoración médico legal em El Departamento de Ucayali. Rev. Diagnóstico. 2011;50(3): 119-22.

3. Ministério da Saúde (BR). Secretaria de Vigilância em Saúde. Departamento de Vigilância de Doenças e Agravos não Transmissíveis e Promoção da Saúde. VIVA- Sistema de Vigilância de Violências e Acidentes, 2008 e 2009. Ministério da Saúde, Brasília, 2010.

4. Secretaria de Saúde (PR). Atenção à mulher em situação de violência. Linha Guia. Versão preliminar. 2012. [acesso em 09 abr 2015]. Disponível: http:// www.saude.pr.gov.br/arquivos/File/Linha_Guia_ Violencia_Sexual_contra_a_Mulher2.pdf

5. Ministério da Saúde (BR). Secretaria de Atenção à Saúde. Departamento de Ações Programáticas Estratégicas. Prevenção e tratamento dos agravos resultantes da violência sexual contra mulheres e adolescentes: norma técnica. 3. ed. Brasília (DF); 2012.

6. Costa DAC, Marques JF, Moreira KAP, Gomes LFS, Henriques ACPT, Fernandes AFC. Assistência multiprofissional à mulher vítima de violência: atuação de profissionais e dificuldades encontradas. Cogitare enferm. 2013;18(2):302-9.

7. Facuri CO, Fernandes AMS, Oliveira KD, Andrade TS, Azevedo RCS. Violência sexual: estudo descritivo sobre as vítimas e o atendimento em um serviço universitário de referência no Estado de São Paulo, Brasil. Cad. Saúde Pública. 2013;29(5):889-98.

8. Sistema de Informação de Agravos de Notificação - SINAN (BR). Ficha de Notificação. Investigação individual. Violência doméstica, sexual e outras violências. [acesso em 14 jan 2015].Disponível: http:// dtr2004.saude.gov.br/sinanweb/novo/Documentos/ SinanNet/fichas/violencia.pdf

9. Center for Disease Control and Prevention (CDC). The National Intimate Partner and Sexual Violence Survey (NISVS):2010 summary report. Atlanta: National Center for Injury Prevention and Control, Centers for Disease Control and Prevention; 2011. [acesso em 09 abr 2015]. Disponível: http://www.cdc.gov/ violenceprevention/nisvs/summary_reports.html

10. Martins CBG, Jorge MHPM. Abuso sexual na infância e adolescência: perfil das vítimas e agressores em município do sul do Brasil. Texto contexto enferm. 2010;19(2):246-55.

11. Souto RQ, Leite CCS, França ISX, Cavalcanti AL. Violência sexual contra mulheres portadoras de necessidades especiais: perfil da vítima e do agressor. 
Cogitare enferm. 2012;17(1):72-7.

12. Costa AM, Moreira KAP, Henriques ACPT, Marques JF, Fernandes AFC. Violência contra a mulher: caracterização de casos atendidos em um centro estadual de referência. Rev Rene. 2011;12(3):627-35.

13. Amarijo CL, Acosta DF, Silva CD, Oliveira Gomes $\mathrm{VLO}$. Factors associated with sexual violence against women: analysis of police reports. Cogitare enferm. 2014;19(4):701-7.

14. Roland ME, Myer L, Martin LJ, Maw A, Batra P, Arend E, et al. Preventing Human Immunodeficiency Virus Infection among sexual assault survivors in Cape Town, South Africa: an observational study. AIDS Behav. 2012;16(4):990-98.

15. Oshikata CT, Bedone AJ, Papa MSF, Santos GB, Pinheiro CD, Kalies AH. Características das mulheres violentadas sexualmente e da adesão ao seguimento ambulatorial: tendências observadas ao longo dos anos em um serviço de referência em Campinas, São Paulo, Brasil. Cad. Saúde Pública. 2011;27(4):701-13.

16. Raimondo ML, Labronici LM, Larocca LM. Retrospecto de ocorrências de violência contra a mulher registradas em uma delegacia especial. Cogitare enferm. 2013;18(1):43-9

17. Chacko L, Ford N, Sbaiti M, Siddiqui R. Adherence to HIV post-exposure prophylaxis in victims of sexual assault: a systematic review and meta-analysis. Sex Transm Infect. 2012;88(5):335-41. 